\title{
Wedge Prism for Direction Resolved Speckle Correlation Interferometry
}

\author{
Chandra S. Vikram \\ University of Alabama in Huntsville \\ Center for Applied Optics \\ Huntsville, Alabama 35899
}

Phone: (256) 890-6030, Ext. 472

Fax: (256) 890-6618

E-mail: vikramc@email.uah.edu

\author{
Martin J. Pechersky \\ Westinghouse Savannah River Company \\ Savannah River Technology Center \\ Aiken, South Carolina 29802
}

\begin{abstract}
The role of a wedge prism for strain sign determination and enhancing the sensitivity for sub-fringe changes is emphasized. The design and incorporation aspects for in-plane sensitive interferometers have been described in detail. Some experimental results dealing with stress determination by laser annealing and speckle correlation interferometry are presented. The prism can also be applied to produce standardized carrier fringes in spatial phase shifting interferometry.
\end{abstract}

Subject terms: speckle interferometry; wedge prism; strain sign; residual stress; spatial phase shifting; carrier fringes; bi-axial interferometer; small strains.

\section{Introduction}

Speckle pattern interferometry ${ }^{1-5}$ is now a well established technique for the strain analysis. A particular version; speckle correlation interferometry using two beams to symmetrically illuminate the object is very popular for in-plane strain analysis along a particular direction. Usually the magnitude of the strain is obtained between the two image storages before and after the deformation. The issue of direction (positive or negative) is generally not addressed. In a large number of practical situations and controlled laboratory experiments, the direction is already known and hence the reduced need to address the issue.

On the other hand, the technique is applicable to a number of practical situations where the direction may be unknown and still required for the analysis. In our particular interest, we have successfully demonstrated that local spot heating and subsequent surface strain measurements by speckle correlation interferometry can provide quantitative information about the object stress. ${ }^{6-11}$ Recently Zhang ${ }^{12}$ applied speckle correlation interferometry to 
determine surface strains induced by blind-hole-drilling procedure of the residual stress evaluation. All these experiments have been in laboratory conditions with samples under known stress situations. Nevertheless, the technique has far reaching consequences for insitu applications in a large number of geophysical and engineering structures. In those situations, it will be necessary to obtain the strain direction as well, for example to distinguish between compressive and tensile stress cases.

Although not specially addressed to the sign problem, the fringe carrier method ${ }^{13,14}$ of moving a lens used to divergently illuminate the surface does provide a carrier fringe pattern and can successfully determine the sign of the unknown motion. However, there are limitations to obtain constant fringe frequency. Also, in several quantitative applications, collimating rather than divergent illuminating beams are desired. One may tilt the subject illuminating mirror to produce the carrier fringe and in fact we have been using that technique routinely. However, as our calculation will follow, to obtain a few fringes in a reproducible quantitative fashion would require costly controls particularly if motorized system is desired. On the other hand, a suitably fabricated wedge prism introduced in the subject illuminating path can easily give uniform frequency carrier fringe pattern. In place $180^{\circ}$ rotation of the prism can do the job as well but with double frequency of the fringe pattern.

Another advantage of the carrier approach is to study subfringe changes as the shifts are easier to measure. Obviously, our analysis mode here emphasizes the fringe counting rather than phase map over the surface obtained by temporal phase shifting interferometry. In fact, in the stress measurement problems, we have found that fringe counting along a line yields enough quantitative data. $6,8,10$

In this paper, we present the design aspects of such a prism with some experimental results. Besides the current need of the sign determination, the approach should also be useful in spatial phase shifting interferometry. It is worth noticing here that the common temporal phase shifting interferometry requires several phase shifted frames for subsequent phase calculations. In the spatial version, a carrier fringe pattern modulated by the unknown motion induced shifts is analyzed as such.

Incidentally, in the single-carrier or spatial phase shifting interferometry, ${ }^{13-16}$ the unknown carrier phase determination itself can be a part of the entire computation process - at the cost of substantial computer time. Thus, if possible, precisely known phase of the carrier should be helpful in those applications as well. In our particular application of the residual stress measurements, assuming the analysis is performed by the fringe counting along a particular direction ${ }^{6,8,10}$ without temporal or spatial phase shifting interferometry, the precisely known carrier information becomes very important.

\section{Wedge Prism in Interferometer}

A typical speckle correlation interferometer may be represented by Fig.1. The symmetrical illuminating beam arrangement is shown suitable for in-plane strain analysis along the $\mathrm{x}$ - 
axis. Also included is a wedge prism, which can be rotated in-plane or inserted (or removed) in the path before the second storage. The customary phase difference between the two illuminating beams ( illumination angle $\theta$ each ) is ${ }^{1-5}$

$$
\frac{2 \pi}{\lambda}(x \sin \theta+x \sin \theta),
$$

where two terms represent the contributions due to two symmetrical beams.

Consequently, a correlation fringe corresponds to the motion (along the $\mathrm{x}$-direction) as spacing

$$
u_{x}=\frac{\lambda}{2 \sin \theta}
$$

One may obtain a carrier fringe pattern by tilting one of the object illuminating beams by $\Delta \theta$ resulting in the phase change $2 \pi x \cos \theta \Delta \theta / \lambda$. This corresponds to the correlation fringe frequency of $\Delta \theta \cos \theta / \lambda$. For a nominal frequency of 3 fringes per $\mathrm{cm}$, HeNe laser wavelength of $632.8 \mathrm{~nm}$, and $\theta=45^{\circ}, \Delta \theta$ comes out to be about $.000268474 \mathrm{rad}$ or about $0.0153824 \mathrm{deg}$. If the accuracy of an order of magnitude better is desired for repetitive implementations, motorized stages become very costly, if available. One may always obtain fringes by manual or motorized rotation stages, however for repetitive and motorized applications an option is desired. On the other hand, a suitably fabricated wedge prism, insertion or in-place rotation by $180^{\circ}$ is a rather easy application. This possibility gave us the idea of critically looking into the wedge prism.

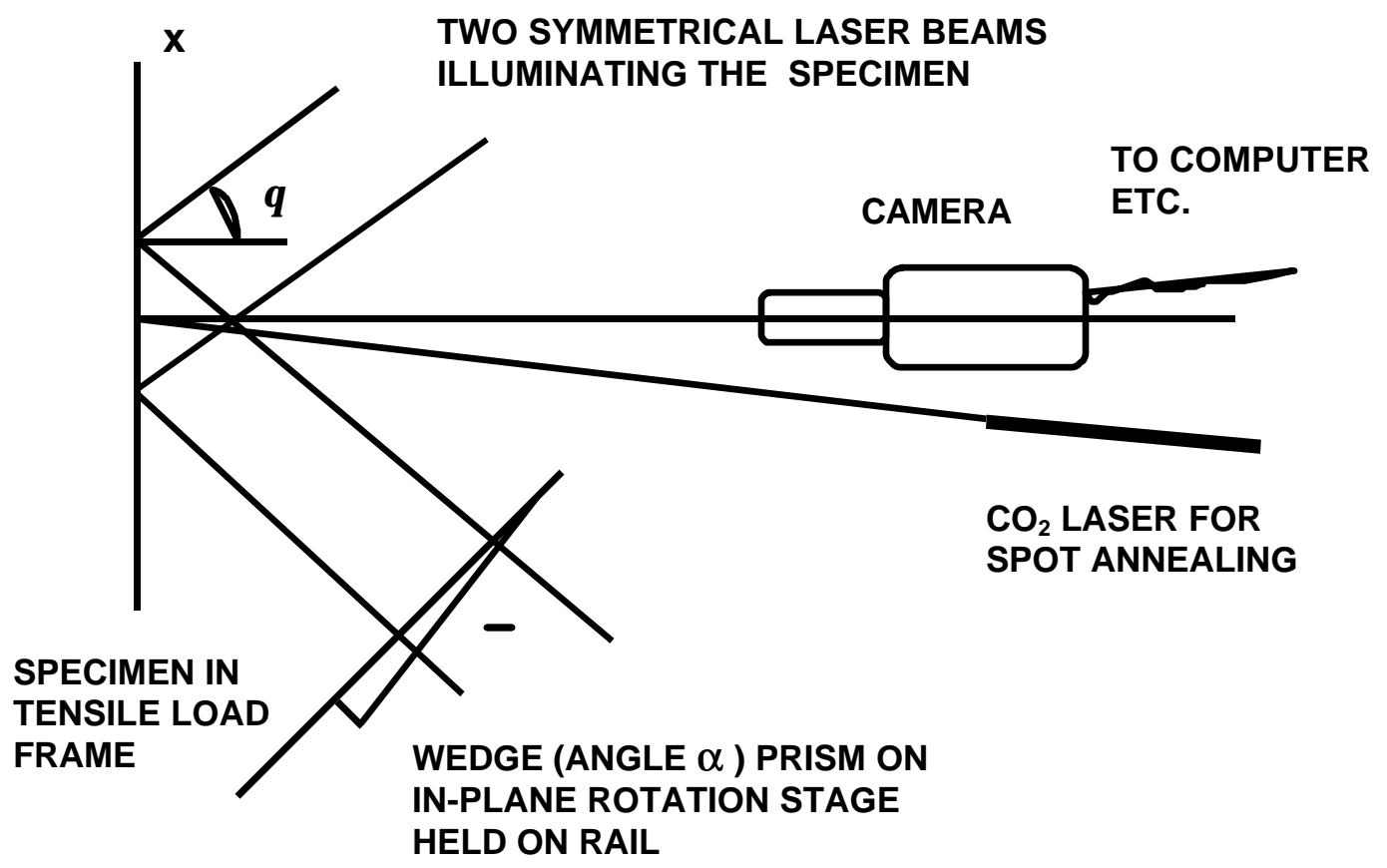


Fig. 1 Schematic diagram showing speckle correlation interferometry set up with symmetrically illuminated beams. Also shown are wedge prism in one of the beam paths and a laser to locally anneal spot on the specimen.

For small angle of incidence, the deviation of a ray incident on the wedged window with wedge angle $\alpha$ is ( $n-1) \alpha$, where $n$ is the refractive index of the material. Thus, the desired wedge angle $\alpha$ for a fringe frequency $v$ is

$$
\alpha=\frac{v \lambda}{(n-1) \cos \theta} \text {. }
$$

For $\lambda=632.8 \mathrm{~nm}, \theta=45^{\circ}, n=1.45702$ ( Fused Silica material), $\alpha$ comes out to be 2.02 arc min for $v$ as 3 fringe/cm. We obtained the custom fabricated ${ }^{17}$ wedges using the following specification:

Window, Special Wedged, $633 \mathrm{~nm}$ usage

Surface figure wavelength/10

Surface quality 10-5 research grade laser polish

Substrate 2 inch diameter $\times 0.375$ inch thick, Fused Silica material

Wedge to be 2 arc min \pm 10 arc second

Wedge difference between one pair to be within 5 arc second

Coated: AR coated both surfaces @ 633 nm, AOI=0 deg

One pair $=$ two windows

Marking: Arrow toward Wedged surface @ higher thickness point

The tolerances can be reduced but at increased cost. The order in pair is for simultaneous use in horizontal and vertical interferometers.

The mechanical aspect of the prism placement can in fact be implemented in two ways. First is to place the prism on a rail and then move it into the laser beam cross-section. This has been the intended mode. The other attractive possibility is to retain the prism in the beam cross-section and just rotate it in its own plane by $180^{\circ}$. In fact we used the identical pair (within tolerance limits) to our advantage. Our dual axis interferometer system, basically composed of two interferometers can independently study in plane strains along horizontal (as seen in Fig.1) and the vertical directions. In the vertical interferometer, a collimated beam illuminates the object area at $45^{\circ}$ angle. Half of the beam is directly illuminating object. Using a mirror in the horizontal plane. the remaining wavefront is reflected back to illuminate the object again to form the other illuminating beam of the interferometer. This kind of interferometer is not common but established. ${ }^{3 \text {, }}$ 
${ }^{18,19}$ Coming back to the wedge prism, one prism is kept in one of the beams of the horizontal interferometer and rotated in plane by $180^{\circ}$ before the second storage. In the vertical interferometer, the prism is just inserted in the entire original illuminating crosssection before the second storage. Now both the illuminating beams are rotated symmetrically by the same amount, we have double the effect. This way, the horizontal and vertical interferometers would give identical carrier fringe frequency. Figure 2 represents the schematics of the process.

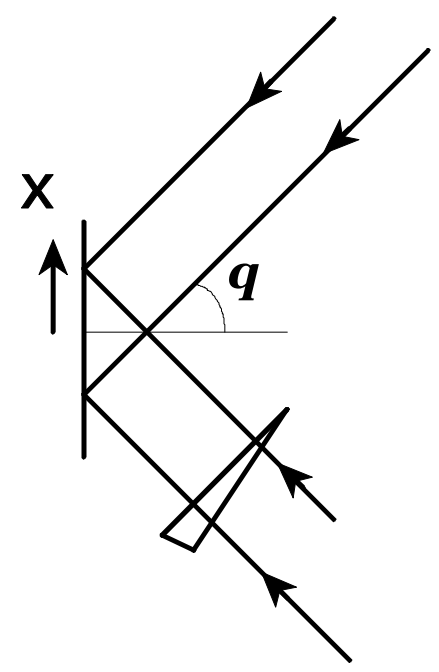

(a)

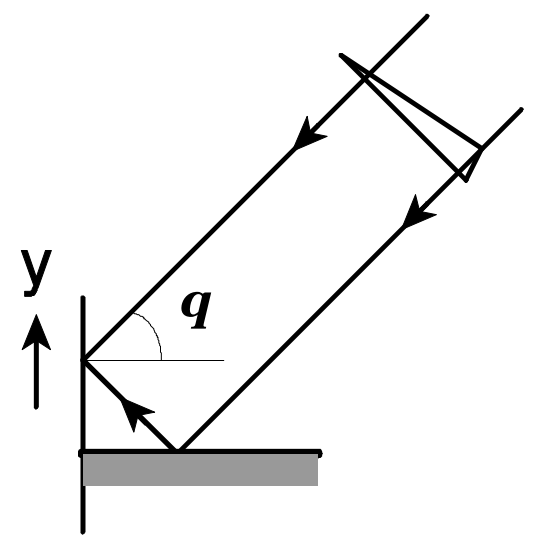

HORIZONTAL INTERFEROMETER

Wedge already present as shown but rotated $180^{\circ}$ in plane before the second storage. The rotation effectively increases the phase along the $\mathrm{x}$-direction. Object motion along the positive $x$ direction in the beam also increases the optical phase. So the fringe frequency will increase (addition of the fringe patterns) for the object motion along $+\mathrm{x}$ direction.

\section{VERTICAL INTERFEROMETER}

Wedge introduced as shown before the second storage in the directly illuminated beam. Positive $(+y)$ direction object motion means reduced optical path for the direct beam to the camera - so does the introduction of the prism. Thus, positive object motion and wedge introduction will increase the fringe frequency.

Fig. 2 Wedge prism incorporation so the positive object motions increase the fringe frequency due to the wedge alone. (a) and (b) represent horizontal and vertical interferometers respectively as described. 
As shown in Fig. 2, the wedges have been incorporated into our dual-axis speckle interferometer. The results are close to those expected by the tolerance limits. Some wedge-induced fringes are shown in the bi-axial stress measurements results (Sec. 3). The fringe frequency $v$ in terms of the wedge angle $\alpha$ being $v=\alpha(n-1) \cos \theta / \lambda$, where $\theta$ $=45^{\circ}, n=1.45702$ and $\lambda=632.8 \mathrm{~nm}$. For our custom-fabricated $\alpha=2 \operatorname{arc} \min . \pm 10$ arc sec. Prism, we obtain $v=(2.97 \pm 0.25)$ fringe $/ \mathrm{cm}$. Thus, if the prism is introduced in one of the illuminating beams before the second storage, we should have $5.94 \pm 0.5$ and $8.02 \pm$ 0.68 fringes along the vertical $(2.0 \mathrm{~cm})$ and horizontal $(2.7 \mathrm{~cm})$ views respectively. However, in our biaxial interferometer, the vertical interferometer is obtained by the wavefront division using a single mirror. Thus, by introducing the wedge in the common incident wavefront, twice the effect is obtained because both the beams of the vertical interferometer will be rotated due to the wedge. To obtain the same effect in the horizontal interferometer (two symmetrical beams are obtained by amplitude division using a cube beam splitter), we placed the wedge in one of the object illuminating beams but rotated in place by $180^{\circ}$ before the second storage. Thus, keeping the double prism effect into account, we should expect $11.88 \pm 1.00$ and $16.04 \pm 1.36$ fringes along the vertical and horizontal views respectively. As we see, the actual interferograms (Sec. 3) show about 13 and 18 fringes respectively; they are close to the tolerance limits of the fabrication process.

\section{Some Experimental Results}

As the process of residual stress measurement using laser speckle interferometry and local laser annealing is well discussed elsewhere, ${ }^{6-11}$ we are reporting the results relevant to the incorporation of the wedge prism. The results are obtained for ASTM ( American Society for Testing and Materials) Standards 304 Stainless steel $(8$ inch $\times 0.5$ inch $\times 0.1$ inch) specimens with yield stress of $45583 \mathrm{lbs}$. Obviously, the specimen cross-section is 0.5 inch $\times 0.1$ inch. A $2 \mathrm{~mm}$ diameter spot on the sample was coated with a temperature sensitive paint of the melting point of $593{ }^{\circ} \mathrm{C}$. The paint highly absorbs the incident $\mathrm{CO}_{2}$ laser power and couples to the steel sample to raise the local surface temperature to $593{ }^{\circ} \mathrm{C}$. At that point, the paint melts and the steel surface becomes mainly reflective again. Such paints not only allow a low power $\mathrm{CO}_{2}$ laser (we use $60 \mathrm{~W}$ ) but to define and control the heated spot side and maximum temperature reached in a practical manner. Further details on the mechanical test frame, the temperature sensitive paint, and the interferometer set up are available elsewhere. ${ }^{6,8}$

Since the room temperature was $22.4{ }^{\circ} \mathrm{C}$, the particular paint allows $570.6{ }^{\circ} \mathrm{C}$ temperature increase in the ramp heating cycle $(5 \mathrm{~s}$ maximum $60 \mathrm{~W}$ at $2.5 \mathrm{~s})$. However, the laser spot size was larger than $2 \mathrm{~mm}$ diameter paint spot, so the entire laser power of $60 \mathrm{~W}$ was not actually used.

Basically an area of $2.7 \mathrm{~cm} \times 2 \mathrm{~cm}$ (including the background each side of the $1.27 \mathrm{~cm}$ wide sample) of the specimen under pretension was stored. A speckle pattern was stored. 
Then the spot was heated as stated above to relieve some stress. After the thermal equilibrium reached, the resulting strains would change the surface. The second storage, subsequent subtraction from that of the first, $3 \times 3$ averaging and the contrast enhancement yield the desired correlation fringes. Finally, a third storage after rotating the wedge
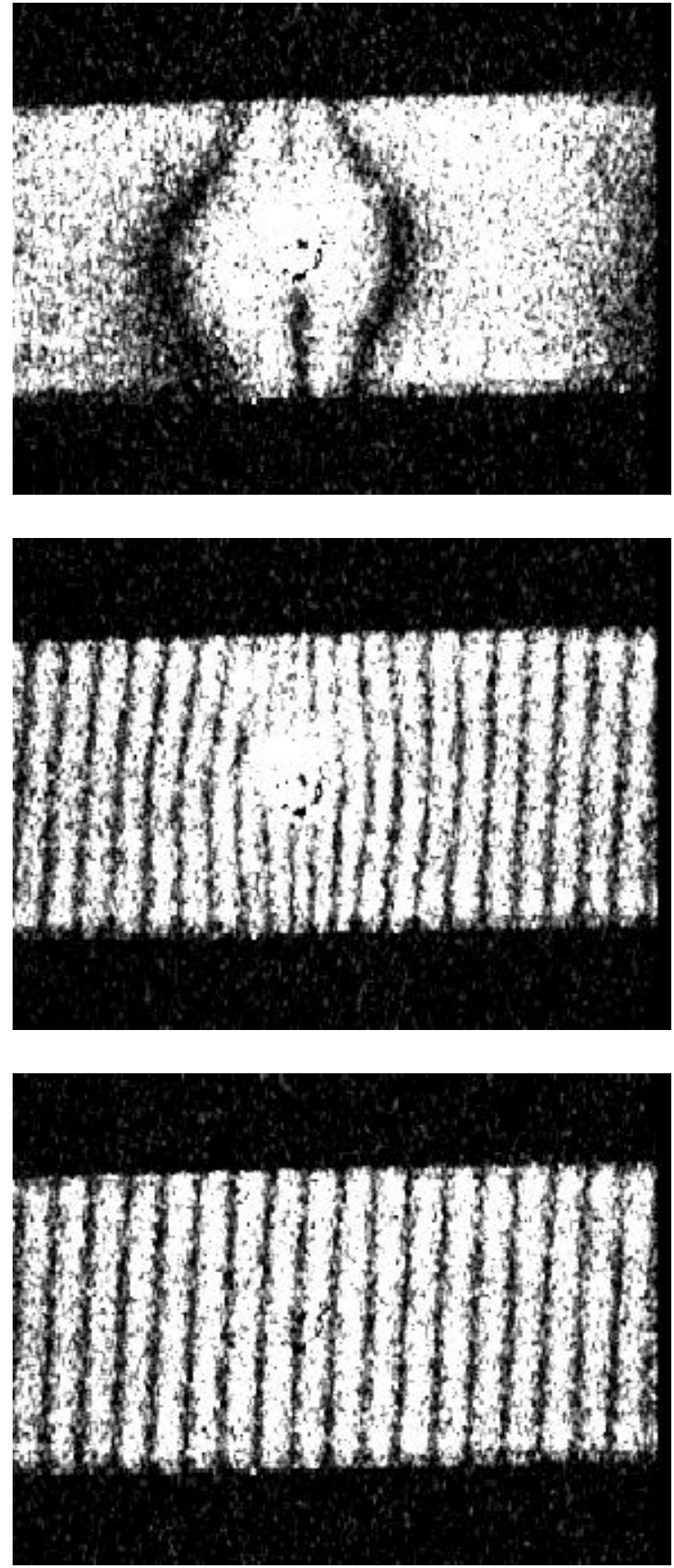

Interferogram with the annealing alone

Interferogram with the wedge and annealing

\section{Interferogram of the wedge alone}

Fig. 3 Interferograms for $80 \%$ pretension case.

prism by $180^{\circ}$ and subsequent subtraction from the first frame leads to the wedge and surface strain effect combined. The difference between the second and the third frame 
obviously yield the wedge fringes alone. However, the wedge-alone fringes can be obtained at any stage just by rotating the wedge by $180^{\circ}$. In our $45^{\circ}$ symmetrically illuminated beams to the surface normal and HeNe laser wavelength of $632.8 \mathrm{~nm}$ wavelength used for interferometry, a fringe corresponds to $0.4475 \mu \mathrm{m}$ in-plane surface displacement. Figure 3 shows the results for $80 \%$ of yield pretension. As we see, the interferogram with wedge alone yields 18 fringes, which is slightly higher from the fabrication requirements $(16.04 \pm 1.36)$. However the 18 fringe count was found to be consistent. 18 in the wedge alone and 21 in the wedge plus annealing situation compares well with about 3 fringes in the annealing alone case. With our direction sensitive interferometer set as shown in Fig. 2, the increase in the number of fringes in the combination shows surface move away from the heated spot - as expected from the sample under tension. Figure 4 shows the results for the sample under $50 \%$ pretension. In this case, the visual guess gives about 1.25 fringes in the annealing case alone. However, wedge alone 18 fringes become 19 in the wedge plus annealing case - clearly showing 1 fringe in the annealing case. Thus strain direction as well as more precision is established.

Regarding the incorporation to the vertical interferometer and to check the double-prism effect as stated in Sec. 2, the prism was incorporated as described in Fig. 2. The expected $11.88 \pm 1$ carrier fringes are close to the actual 13 as shown in Fig. 5. The object was a flat steel specimen covering the entire field of view $(2.7 \mathrm{~cm} \times 2 \mathrm{~cm})$. We have been performing residual stress experiments with sign determination capability with this mode as well. Needless to mention, in place rotation as done in the horizontal interferometer can double the fringe frequency in this case as well. However, since we use identical wedge prisms in both the interferometers, the current procedure yields identical carrier fringe frequency in both the segments. On the other hand, if in place rotation in both the segments still keeping identical fringe frequencies is desired, one may have the prisms fabricated with different (one double the other) wedge angles. The higher angle wedge obviously goes to the horizontal interferometer. 

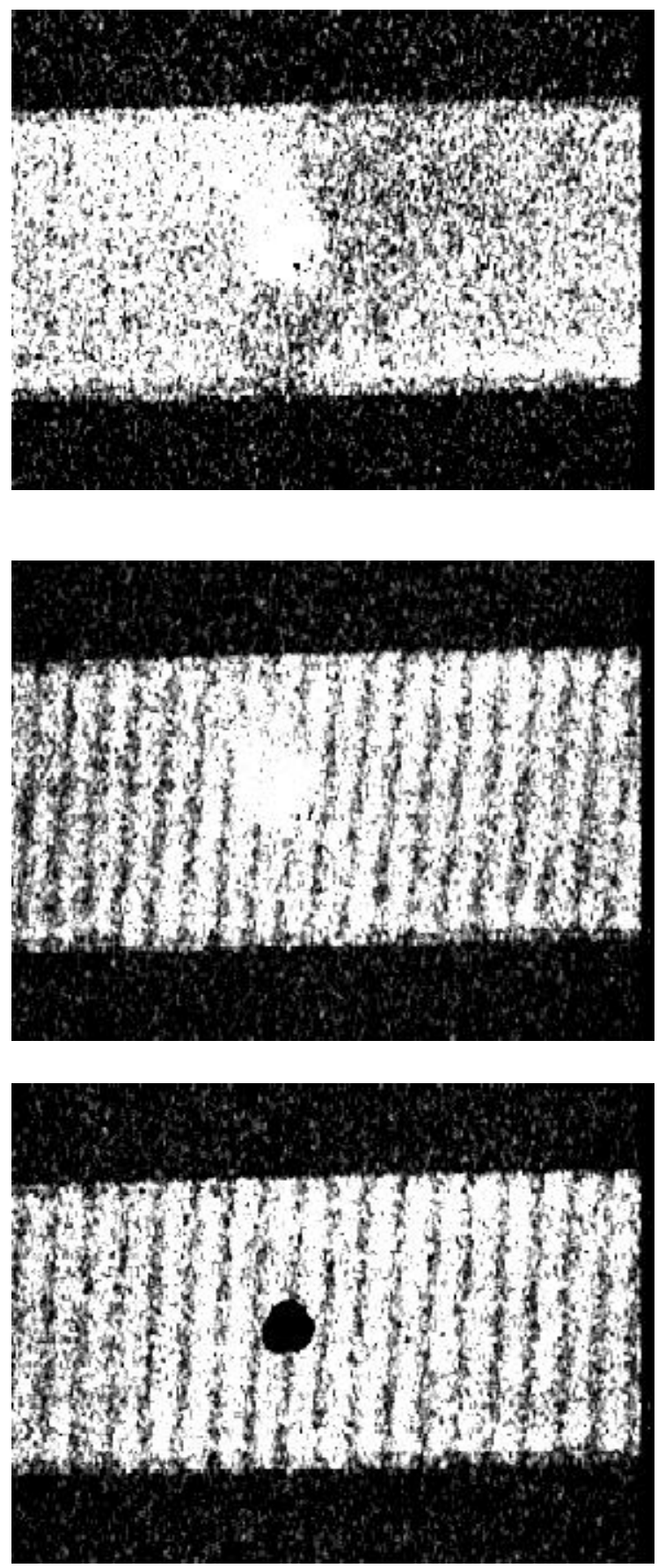

Interferogram with the annealing alone
Interferogram with wedge and annealing

Interferogram of the wedge alone

Fig. 4 Interferograms for $50 \%$ pretension case. 


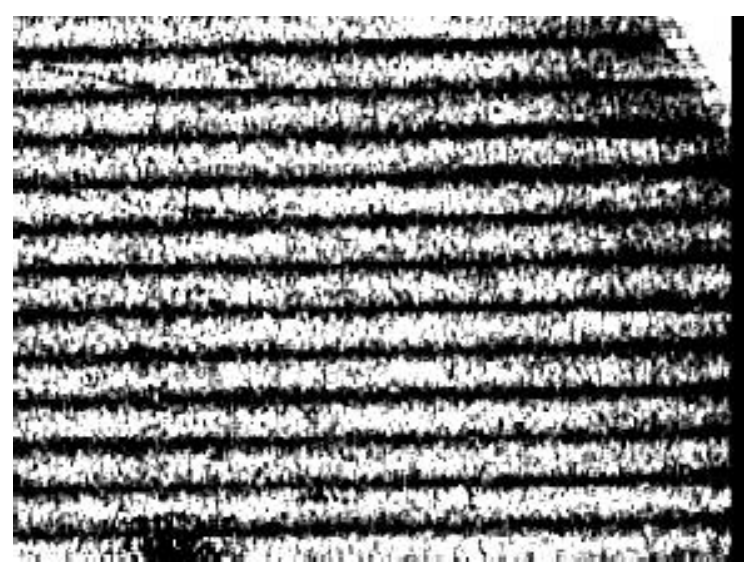

Fig. 5 Correlation fringes due to insertion of the wedge prism in the vertical interferometer.

\section{Discussion}

We have shown the predicable frequency carrier fringes in speckle correlation interferometry can be obtained by introducing a wedge prism in the illumination beam path. The prism can be inserted before the second storage or just in place rotated. Once the prism is fabricated to specifications, the repetitive application can be simple without very elaborate controls. The carrier fringes can readily yield the sense of the strain direction. Also, in case of sub-fringe strains, the measurement sensitivity is enhanced as the fringe shift is easy to measure. Finally, for spatial phase-shifting interferometry applications, the prism can be a standardized mode of providing the carrier fringes.

\section{Acknowledgment}

This document was prepared in conjunction with work performed by Westinghouse Savannah River Company for the Department of Energy under contract number DEAC09-96SR18500.

\section{References}

1. R. K. Erf, Ed., Speckle Metrology, Academic Press, New York (1978).

2. J. C. Dainty, Ed., Laser Speckle and Related Phenomena, $2^{\text {nd }}$ enlarged ed., SpringerVerlag, Berlin (1984). 
3. R. Jones and C. Wykes, Eds., Holographic and Speckle Interferometry, Second Enlarged Edition, Cambridge University Press, Cambridge (1989).

4. R. S. Sirohi, Ed., Speckle Metrology, Marcel Dekker, New York (1993).

5. C. Joenathan, "Speckle photography, shearography, and ESPI," Chap. 6 in Optical Measurement Techniques and Applications, P. K. Rastogi, Ed., pp. 151-182, Artech House, Boston (1997).

6. M. J. Pechersky, R. F. Miller, and C. S. Vikram, "Residual stress measurements with laser speckle correlation interferometry and local heat treating," Opt. Eng. 34(10), 2964-2971 (1995).

7. M. J. Pechersky, "Method for measuring residual stresses in materials by plastically deforming the materials and interference pattern," U. S. Patent No. 5,432,595, July 11, 1995.

8. C. S. Vikram, M. J. Pechersky, C. Feng and D. Engelhaupt, "Residual-stress analysis by local laser heating and speckle-correlation interferometry," Exp. Techniques 20(6), 27-30 (1996).

9. J. E. Millerd, J. D. Trolinger, C. S. Vikram, and M. J. Pechersky, "Residual stress measurements by laser annealing and speckle interferometry," in Nondestructive Evaluation of Aircraft, Airports, and Aerospace Hardware, R. D. Rempt and A. L. Broz, Eds., Proc. SPIE 2945, 78-86 (1996).

10. M. J. Pechersky and C. S. Vikram, "Determination of residual stresses by local annealing and laser speckle pattern interferometry," in Post Conference Proceedings of the 1997 SEM Spring Conference on Experimental Mechanics, June 2-4, 1997 Bellevue, Washington, 116-120 (1997).

11. M. J. Pechersky, P. S. Lam, and C. S. Vikram, "Bi-directional residual stress measurements with laser annealing and speckle interferometry," in Proceedings of the SEM Spring Conference on Experimental Mechanics and Applied Mechanics and Experimental/Numerical Mechanics in Electronic Packaging III, Houston, Texas, June 1-3, 1998, pp. 184-186 (1998).

12. J. Zhang, "Two-dimensional in-plane electronic speckle pattern interferometer and its application to residual stress determination," Opt. Eng. 37(8), 2402-2409 (1998).

13. A. Fernandez, J. Blanco-Garcia, A. F. Doval, J. Bugarin, B. V. Dorrio, C. Lopez, J. M. Alen, M. Perez-Amor, and J. L. Fernandez, "Transient deformation measurements by double-pulsed-subtraction TV holography and the Fourier transform method," Appl. Opt. 37(16), 3440-3446 (1998).

14. A. Davila, G. H. Kaufman, and C. Perez-Lopez, "Transient deformation analysis by a carrier method of pulsed electronic speckle-shearing pattern interferometry," Appl. Opt. 37(19), 4116-4122 (1998).

15. J. Gu and F. Chen, "Fast Fourier transform, iteration, and least-squares-fit demodulation image processing for analysis of single-carrier fringe pattern," J. Opt. Soc. Am. A 12(10), 2159-2164 (1995).

16. P. H. Chan, P. J. Bryanston-Cross, and S. C. Parker, "Fringe-pattern analysis using a spatial phase-stepping method with automatic phase unwrapping," Meas. Sci. Technol. 6(9), 1250-1259 (1995).

17. JANOS Technology, Inc., HCR \#33, Box 25, Route 35, Townshend, VT 05353-7702. 
18. R. Jones and J. A. Leendertz, "Elastic constant and strain measurements using a three beam speckle pattern interferometer," J. Phys. E. 7(8), 653-657 (1974).

19. R. Jones, "The design and application of a speckle pattern interferometer for total plane strain field measurement," Optics and Laser Technology 8(5), 215-219(1976) 